\title{
Alcohol Moiety-Tethering Acylazides as Versatile Isocyanate Precursors for Polymerization Initiators and Monomers
}

Yosuke Akae ${ }^{1,2 *}$, Masayoshi Sakurai ${ }^{1}$, and Toshikazu Takata ${ }^{1,3 *}$

${ }^{1}$ School of Materials and Chemical Technology, Tokyo Institute of Technology, Nagatsuta-cho 4259, Midori-ku, Yokohama 226-8503, Japan

${ }^{2}$ Research Fellow of Japan Society for the Promotion of Science

${ }^{3}$ Graduate School of Advanced Science and Engineering, Hiroshima University, Higashi-Hiroshima, 7398527, Japan

E-mail: takatats@hiroshima-u.ac.jp (T. Takata). 
Table of Contents:

1. General Methods $\quad$ S3

2. Chemical Synthesis $\quad$ S4-S10

3. Spectra of synthesized compounds $\quad$ S11-S13

4. References $\quad$ S14 


\section{General Methods}

${ }^{1} \mathrm{H}-(400 \mathrm{MHz})$ NMR spectra were recorded on a JEOL AL-400 spectrometer and ${ }^{1} \mathrm{H}-(300 \mathrm{MHz}) \mathrm{NMR}$ spectra were recorded on a Bruker DPX spectrometer 300 using $\mathrm{CDCl}_{3}$ and DMSO- $d_{6}$ as the solvents, calibrated using residual undeuterated solvent or tetramethylsilane as the internal standard. IR spectra were recorded on a JASCO FT/IR-230 spectrometer. ESI HR-MS spectra were taken on a Bruker Daltonics micrOTOF II at the Center for Advanced Material Analysis, Tokyo Institute of Technology on request. MALDI-TOF MS were measured with a Shimazdu AXIMA-CFR mass spectrometer using dithranol as matrix. Analytical GPC was performed at $30{ }^{\circ} \mathrm{C}$ in $\mathrm{CHCl}_{3}(0.85 \mathrm{~mL} / \mathrm{min})$ using a JASCO PU-2080 system equipped with a set of Shodex K-804 and Shodex K-805 columns, and in dimethylformamide (DMF) (5 $\mathrm{mM} \mathrm{LiBr}, 0.85 \mathrm{~mL} / \mathrm{min}$ ) using a JASCO PU-2080 system equipped with a set of TOSOH TSKgel G2500H and $\mathrm{G} 4000 \mathrm{H}$ columns. The number average molecular weight $\left(M_{\mathrm{n}}\right)$, weight average molecular weight $\left(M_{\mathrm{w}}\right)$, and polydispersity index $\left(M_{\mathrm{w}} / M_{\mathrm{n}}\right)$ of the polymers were calculated on the basis of a polystyrene calibration. Wako Gel ${ }^{\circledR}$ C-400HG (Wako Pure Chemical Industries) was used for silica gel column chromatography. Cerite $^{\circledR}$ (Wako Pure Chemical Industries) was used for removal of metal compounds. $\delta$-Valerolactone and $\mathrm{CH}_{2} \mathrm{Cl}_{2}$ was purchased from Tokyo Chemical Industry Company and distilled before using. Other commercially available reagents and solvents were used without further purification unless otherwise noted.

All compounds given below bear the same formula numbers as used in the main text. Compounds unlabeled in the main text are labeled with letters $[\mathbf{S 1}-\mathbf{S 3}]$. 


\section{Chemical Synthesis}

Synthesis of $1^{1,2)}$

$\mathrm{N}_{\mathrm{CH}}^{\mathrm{O}}$

1

Diphenylphosphorylazide $(6.1 \mathrm{~g}, 22 \mathrm{mmol})$ was added to a solution of 4-hydroxymethyl benzoicacid acid (3.0 g, 20 mmol) and $\mathrm{Et}_{3} \mathrm{~N}(2.2 \mathrm{~g}, 22 \mathrm{mmol})$ in $\mathrm{CH}_{2} \mathrm{Cl}_{2}(50 \mathrm{~mL})$, then, the mixture was stirred at room temperature for 2 hours. To the mixture, sat. $\mathrm{NH}_{4} \mathrm{Cl}$ aq. was added, extracted with $\mathrm{CH}_{2} \mathrm{Cl}_{2}$, washed with water, and purified by silica gel column chromatography (eluent; $\left.\mathrm{CH}_{2} \mathrm{Cl}_{2}\right)$ to give the corresponding acyl azide $\mathbf{1}(2.8 \mathrm{~g}, 78 \%)$ as a white solid; ${ }^{1} \mathrm{H}$ NMR (400 MHz, $\left.298 \mathrm{~K}, \mathrm{CDCl}_{3}\right) \delta 8.02(\mathrm{~d}, J=8.6 \mathrm{~Hz}, 2 \mathrm{H}), 7.46(\mathrm{~d}, J=8.6 \mathrm{~Hz}, 2 \mathrm{H}), 4.79(\mathrm{~s}, 2 \mathrm{H}) \mathrm{ppm}$; IR (NaCl) $v$ 3239, 2849, 2359, 2342, 2178, 2133, 1690, 1607, 1573, 1508, 1439, 1416, 1369, 1332, 1271, 1245, 1182, 1132, 1038, 1019, 993, 850, 828, 813, 797, 732, $679 \mathrm{~cm}^{-1}$; ESI-TOF MS (m/z): calc'd for $\mathrm{C}_{8} \mathrm{H}_{7} \mathrm{~N}_{3} \mathrm{NaO}_{2}{ }^{+}\left[\mathrm{M}^{+}\right]$200.0430; found 200.0429. The spectra data of $\mathbf{1}$ is also available for another publication. ${ }^{2)}$

\section{Synthesis of $\mathbf{P 2}^{3)}$}
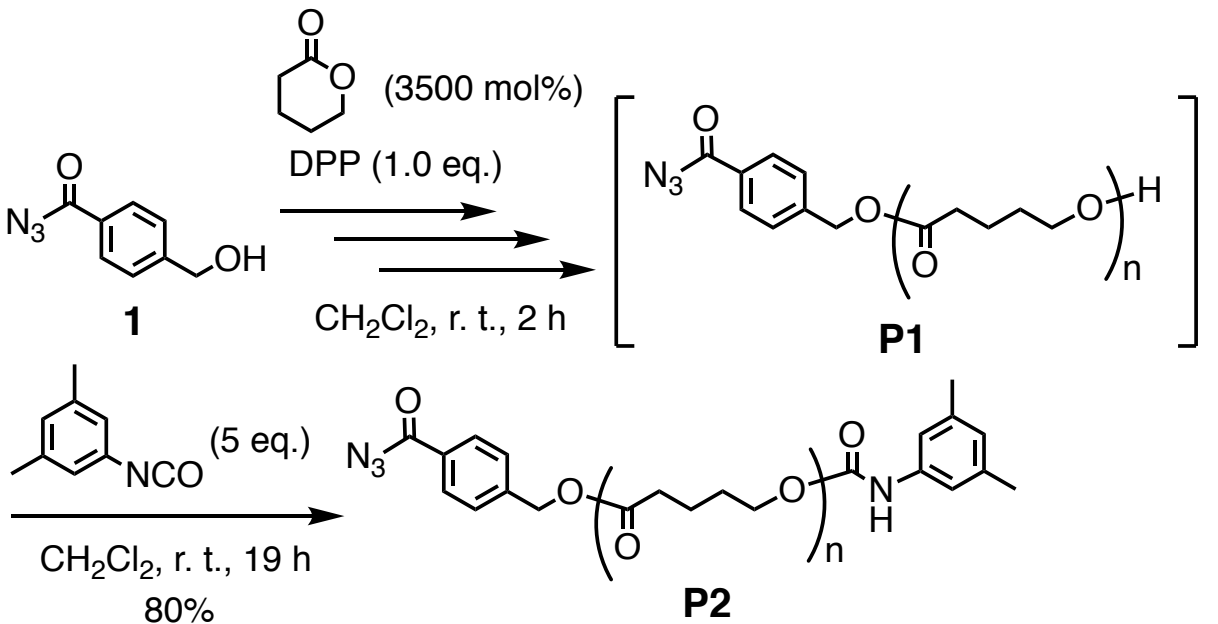

Diphenylphosphate $(0.25 \mathrm{~g}, 1.0 \mathrm{mmol})$ was added to a solution of $1(0.18 \mathrm{~g}, 1.0 \mathrm{mmol})$ and $\delta$-valerolactone $(3.5 \mathrm{~g}$, $35 \mathrm{mmol})$ in $\mathrm{CH}_{2} \mathrm{Cl}_{2}(4.0 \mathrm{~mL})$, then the mixture was stirred for $2 \mathrm{~h}$ at room temperature. 3,5dimethylphenylisocyanate $(0.75 \mathrm{~g}, 5.0 \mathrm{mmol})$ was added to the reaction mixture, and the mixture was stirred for 19 $\mathrm{h}$ at room temperature. The resulting mixture was poured into ethanol $/$ hexane $=1 / 9(\mathrm{v} / \mathrm{v})$ and collected the precipitate. The residue was dried in vacuo to give $\mathbf{P 2}(3.0 \mathrm{~g}, 80 \%)$ as a white solid; $M_{\mathrm{n}, \mathrm{NMR}} 3700 \mathrm{~g} / \mathrm{mol} ; M_{\mathrm{w}} / M_{\mathrm{n}} 1.1 ;{ }^{1} \mathrm{H}$ NMR $\left(400 \mathrm{MHz}, 298 \mathrm{~K}, \mathrm{CDCl}_{3}\right) \delta 8.03(\mathrm{~d}, J=8.4 \mathrm{~Hz}, 2 \mathrm{H}), 7.43(\mathrm{~d}, J=8.4 \mathrm{~Hz}, 2 \mathrm{H}), 7.02(\mathrm{~s}, 2 \mathrm{H}), 6.72-6.69$ (m, 2H), 5.18 $(\mathrm{s}, 2 \mathrm{H}), 4.16\left(\mathrm{t}, 2 \mathrm{H}, J=5.7 \mathrm{~Hz},-\mathrm{CH}_{2} \mathrm{O}-\right.$ of polymer ends), $4.08\left(\mathrm{t},(2 \times \mathrm{n}) \mathrm{H}, J=5.9 \mathrm{~Hz},-\mathrm{CH}_{2} \mathrm{O}-\right), 2.44(\mathrm{t}, 2 \mathrm{H}, J=$ $7 \mathrm{~Hz},-\mathrm{CO}-\mathrm{CH}_{2}-$ of polymer ends), $2.34\left(\mathrm{t},(2 \times \mathrm{n}) \mathrm{H}, J=7 \mathrm{~Hz},-\mathrm{COCH}_{2}-\right), 2.29(\mathrm{~s}, 6 \mathrm{H}), 1.77-1.58(\mathrm{~m},\{(2 \times \mathrm{n})+(2$ $\times$ n) $\} \mathrm{H},-\mathbf{C H}_{2} \mathrm{CH}_{2} \mathrm{O}-$ and $\left.-\mathrm{COCH}_{2} \mathbf{C H}_{2}-\right)$ ppm; IR (NaCl) $v 3441,3356,2957,2893,2874,2358,2342,2174,2138$, $1729,1615,1558,1507,1473,1458,1423,1401,1383,1326,1259,1179,1105,1066,1047,992,954,915,842$, $808,740,690,669 \mathrm{~cm}^{-1}$.

\section{Synthesis of P3}

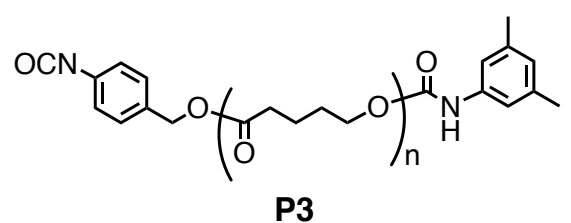


P2 $(0.42 \mathrm{~g}, 0.11 \mathrm{mmol})$ was dissolved in toluene $(2 \mathrm{~mL})$ and stirred at $100{ }^{\circ} \mathrm{C}$ for 2 hours. Solvent was removed under low pressure, and the residue was dried in vacuo to give $\mathbf{P 3}$ (0.42 g, quant) as a white solid; ${ }^{1} \mathrm{H}$ NMR (400 $\left.\mathrm{MHz}, 298 \mathrm{~K}, \mathrm{CDCl}_{3}\right) \delta 7.31(\mathrm{~d}, J=8.6 \mathrm{~Hz}, 2 \mathrm{H}), 7.09(\mathrm{~d}, J=8.6 \mathrm{~Hz}, 2 \mathrm{H}), 7.02(\mathrm{~s}, 2 \mathrm{H}), 6.72-6.69(\mathrm{~m}, 2 \mathrm{H}), 5.08(\mathrm{~s}$, $2 \mathrm{H}), 4.16\left(\mathrm{t}, 2 \mathrm{H}, J=5.7 \mathrm{~Hz},-\mathrm{CH}_{2} \mathrm{O}-\right.$ of polymer ends), $4.08\left(\mathrm{t},(2 \times \mathrm{n}) \mathrm{H}, J=5.9 \mathrm{~Hz},-\mathrm{CH}_{2} \mathrm{O}-\right), 2.42-2.26(\mathrm{~m},(2 \times$ n) $\mathrm{H},-\mathrm{CO}-\mathrm{CH}_{2}-$ of polymer), $2.29(\mathrm{~s}, 6 \mathrm{H}), 1.77-1.58\left(\mathrm{~m},\{(2 \times \mathrm{n})+(2 \times \mathrm{n})\} \mathrm{H},-\mathbf{C H}_{2} \mathrm{CH}_{2} \mathrm{O}-\right.$ and $\left.-\mathrm{COCH}_{2} \mathbf{C H}_{2}-\right)$ ppm; IR (NaCl) v3441, 3356, 2957, 2894, 2874, 2358, 2342, 2265, 1732, 1653, 1615, 1540, 1473, 1458, 1423, 1402 , $1383,1326,1260,1220,1174,1105,1066,1047,954,915,842,808,739,690,669 \mathrm{~cm}^{-1}$. P3_2.6 was also synthesized according to this procedure.

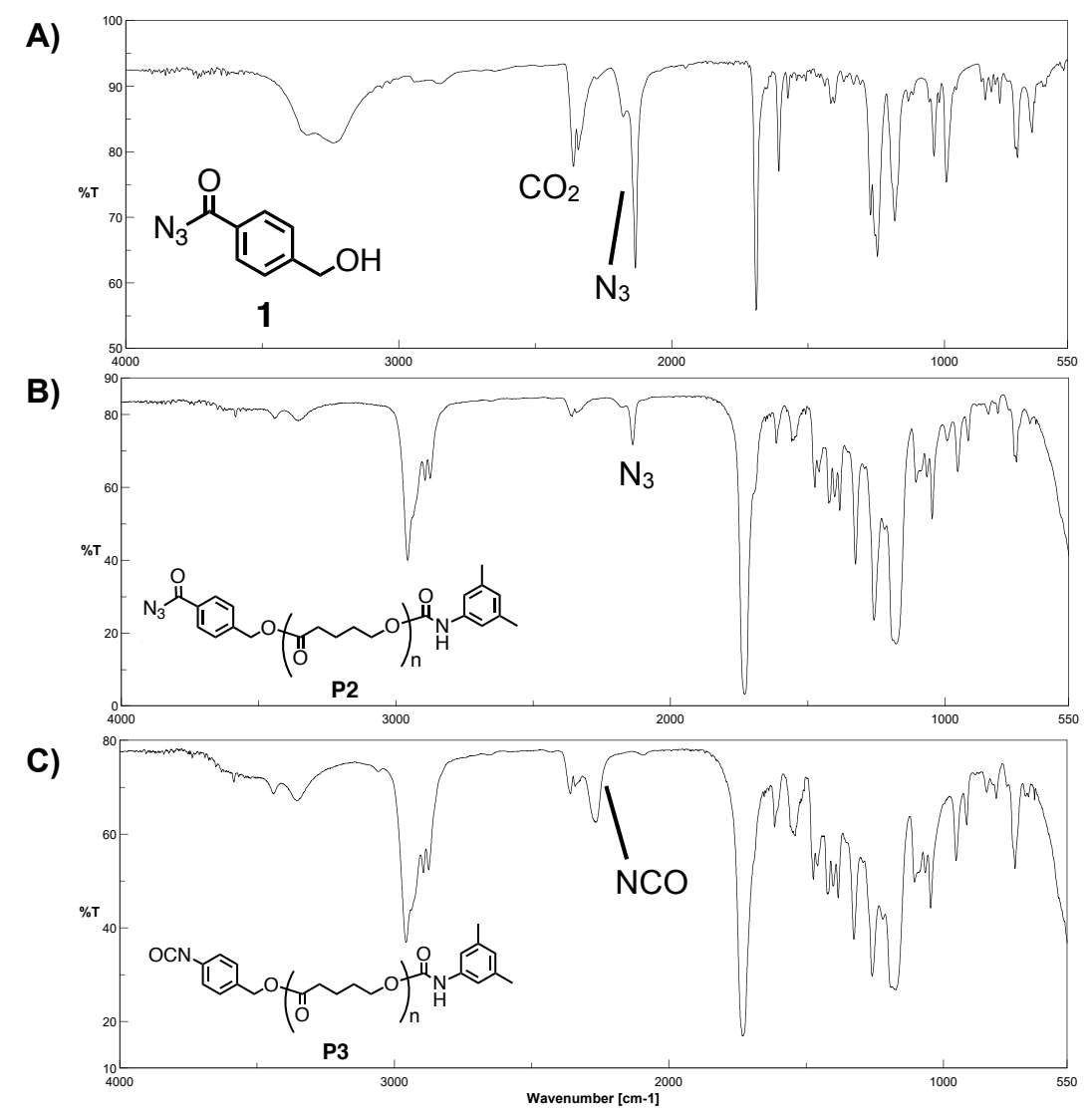

Figure S1. IR spectra of (A) 1, (B) P2, and (C) P3 (NaCl).

\section{Synthesis of P4}<smiles>Cc1cc(C)cc(NC(=O)C(C)(C)CCCCC(=O)OCc2ccc(NC(=O)NCc3ccccc3)cc2)c1</smiles>

2-phenylethylamine (10 mg, $0.080 \mathrm{mmol})$ was added to the solution of $\mathbf{P 3}(0.29 \mathrm{~g}, 0.080 \mathrm{~mol})$ in $\mathrm{CH}_{2} \mathrm{Cl}_{2}(1.0 \mathrm{~mL})$, and the mixture was stirred at room temperature for 18 hours. The resulting mixture was poured into $\mathrm{MeOH}$ and collected the precipitate. The residue was dried in vacuo to give $\mathbf{P 4}(0.26 \mathrm{~g}, 86 \%)$ as a white solid; ${ }^{1} \mathrm{H}$ NMR $(400$ $\left.\mathrm{MHz}, 298 \mathrm{~K}, \mathrm{CDCl}_{3}\right) \delta 7.42-7.37(\mathrm{~m}, 2 \mathrm{H}), 7.32-7.18(\mathrm{~m}, 8 \mathrm{H}), 7.02(\mathrm{~s}, 2 \mathrm{H}), 6.72-6.69(\mathrm{~m}, 2 \mathrm{H}), 6.51(\mathrm{~s}, 1 \mathrm{H}), 5.05(\mathrm{~s}$, $2 \mathrm{H}), 4.16\left(\mathrm{t}, 2 \mathrm{H}, J=5.7 \mathrm{~Hz},-\mathrm{CH}_{2} \mathrm{O}-\right.$ of polymer ends), $4.08\left(\mathrm{t},(2 \times \mathrm{n}) \mathrm{H}, J=5.9 \mathrm{~Hz},-\mathrm{CH}_{2} \mathrm{O}-\right), 3.53(\mathrm{~m}, 2 \mathrm{H}), 2.85$ $(\mathrm{t}, J=7.0 \mathrm{~Hz}, 2 \mathrm{H}), 2.42-2.26\left(\mathrm{~m},(2 \times \mathrm{n}) \mathrm{H},-\mathrm{CO}-\mathrm{CH}_{2}-\right.$ of polymer $), 2.29(\mathrm{~s}, 6 \mathrm{H}), 1.77-1.58(\mathrm{~m},\{(2 \times \mathrm{n})+(2 \times \mathrm{n})\} \mathrm{H}$,

$-\mathrm{CH}_{2} \mathrm{CH}_{2} \mathrm{O}-$ and $\left.-\mathrm{COCH}_{2} \mathbf{C H}_{2}-\right)$ ppm. 


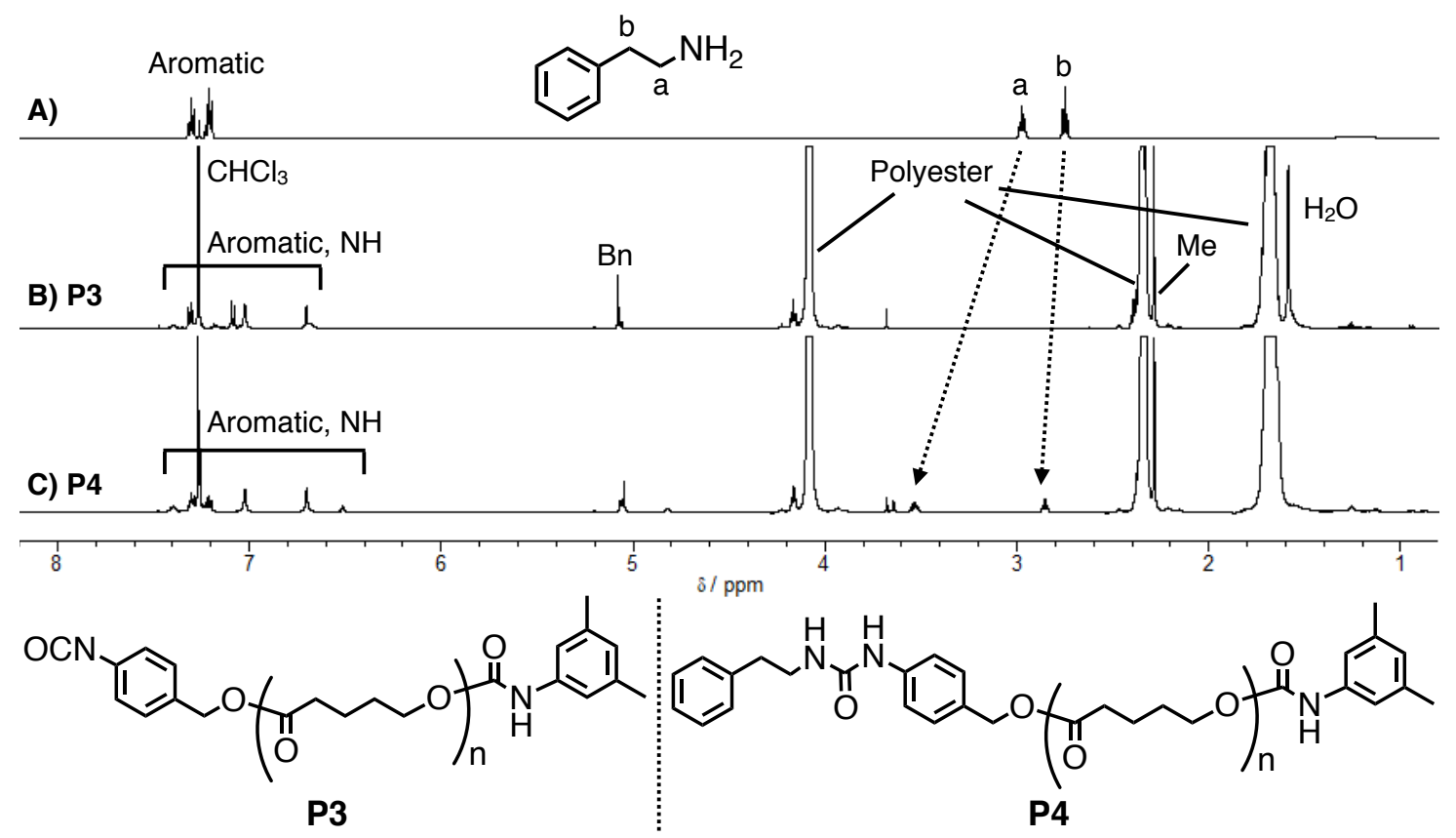

Figure S2. ${ }^{1} \mathrm{H}$ NMR spectra of (A) 2-phenylethylamine, (B) P3, and (C) $\mathbf{P 4}\left(400 \mathrm{MHz}, \mathrm{CDCl}_{3}, 298 \mathrm{~K}\right.$ ).

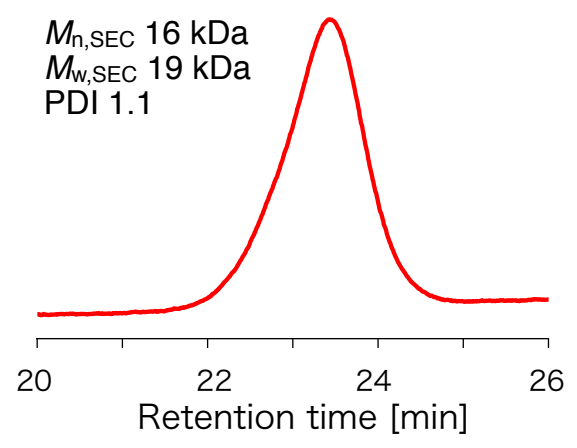

Figure S3. SEC profile of $\mathbf{P 4}$ (eluent; $\mathrm{CHCl}_{3}$; flow rate: $2 \mathrm{~mL} / \mathrm{min}$; detected by RI). 

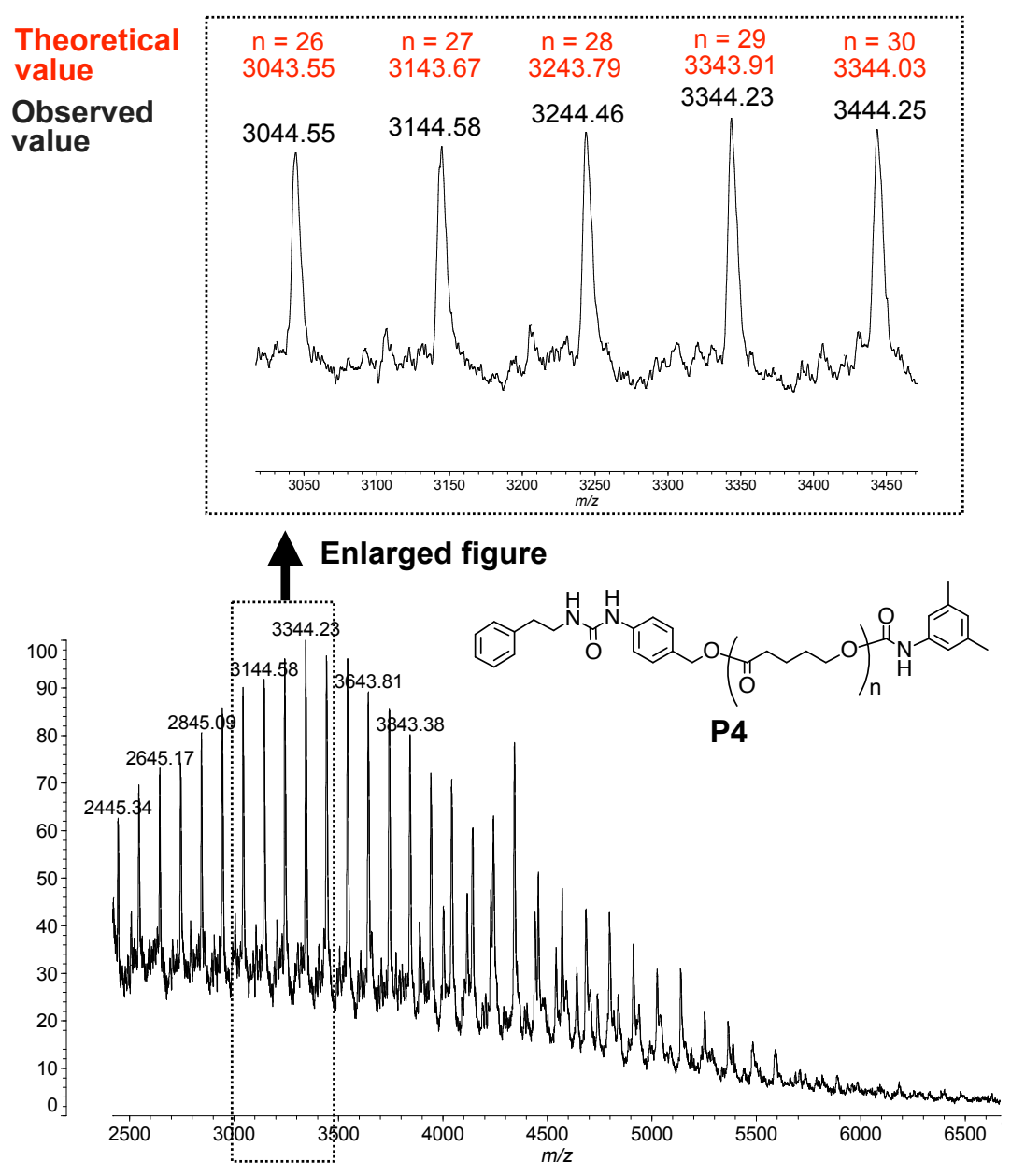

Figure S4. MALDI-TOF-MS of P4 (matrix; dithranol).

\section{Synthesis of P5}

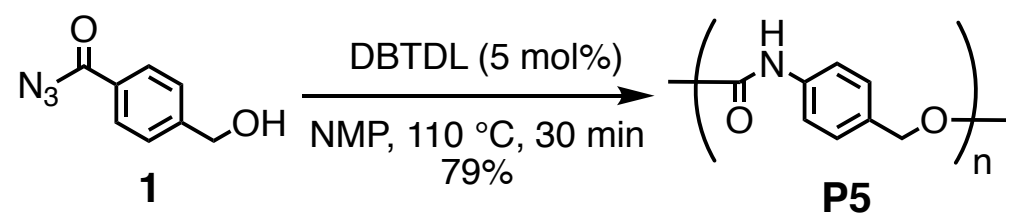

Dibutyltin dilaurate $(54 \mathrm{mg}, 0.085 \mathrm{mmol})$ was added to a solution of $1(0.31 \mathrm{~g}, 1.7 \mathrm{mmol})$ in NMP $(1.7 \mathrm{~mL})$, then the mixture was stirred for $30 \mathrm{~min}$ at $110^{\circ} \mathrm{C}$. The resulting mixture was poured into $\mathrm{MeOH}$ and collected the precipitate. The residue was dried in vacuo to give $\mathbf{P 5}(0.25 \mathrm{~g}, 79 \%)$ as a light yellow solid; $M_{\mathrm{n}, \mathrm{SEC}} 6600 \mathrm{~g} / \mathrm{mol} ; M_{\mathrm{w}} / M_{\mathrm{n}} 2.4 ;{ }^{1} \mathrm{H}$ NMR (300 MHz, 298 K, DMSO-d $)_{6} \delta 9.80(\mathrm{~s}, 1 \mathrm{H}), 7.47$ (d, J=7.5 Hz, 2H), $7.34(\mathrm{~d}, J=7.5 \mathrm{~Hz}, 2 \mathrm{H}), 5.05(\mathrm{~s}, 2 \mathrm{H})$ ppm. 


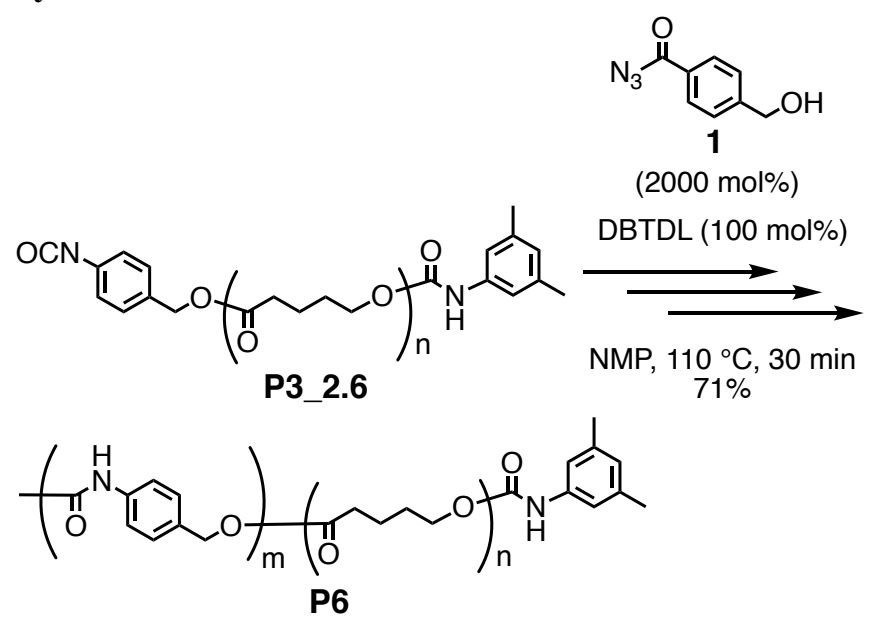

Dibutyltin dilaurate $(54 \mathrm{mg}, 0.085 \mathrm{mmol})$ was added to a solution of $\mathbf{1}(0.31 \mathrm{~g}, 1.7 \mathrm{mmol})$ and P3_2.6 (0.22 g, 0.085 mmol) in NMP $(1.7 \mathrm{~mL})$, then the mixture was stirred for $30 \mathrm{~min}$ at $110{ }^{\circ} \mathrm{C}$. The resulting mixture was poured into $\mathrm{CHCl}_{3}$ and collected the precipitate. The residue was dried in vacuo to give $\mathbf{P 6}(0.38 \mathrm{~g}, 71 \%)$ as a light yellow solid; $M_{\mathrm{n}, \mathrm{SEC}} 9900 \mathrm{~g} / \mathrm{mol} ; M_{\mathrm{w}} / M_{\mathrm{n}} 2.4 ;{ }^{1} \mathrm{H}$ NMR $\left(300 \mathrm{MHz}, 298 \mathrm{~K}, \mathrm{DMSO}-\mathrm{d}_{6}\right) \delta 9.80(\mathrm{~s},(1 \times \mathrm{m}) \mathrm{H}), 7.47(\mathrm{~d}, J=8.5 \mathrm{~Hz},(2$ $\times \mathrm{m}) \mathrm{H}), 7.34(\mathrm{~d}, J=8.5 \mathrm{~Hz},(2 \times \mathrm{m}) \mathrm{H}), 7.07(\mathrm{~s}, 2 \mathrm{H}), 6.55-6.45(\mathrm{~m}, 2 \mathrm{H}), 5.05(\mathrm{~s},(2 \times \mathrm{n}) \mathrm{H}), 3.99(\mathrm{t},(2 \times \mathrm{n}) \mathrm{H}, J=$ $\left.5.5 \mathrm{~Hz},-\mathrm{CH}_{2} \mathrm{O}-\right), 2.31\left(\mathrm{t},(2 \times \mathrm{n}) \mathrm{H}, J=6.6 \mathrm{~Hz},-\mathrm{COCH}_{2}-\right), 2.20(\mathrm{~s}, 6 \mathrm{H}), 1.65-1.44(\mathrm{~m},\{(2 \times \mathrm{n})+(2 \times \mathrm{n})\} \mathrm{H},-$ $\mathbf{C H}_{2} \mathrm{CH}_{2} \mathrm{O}-$ and $\left.-\mathrm{COCH}_{2} \mathbf{C H}_{2}-\right)$ ppm.

Synthesis of 2 was carried out according to Scheme S1.

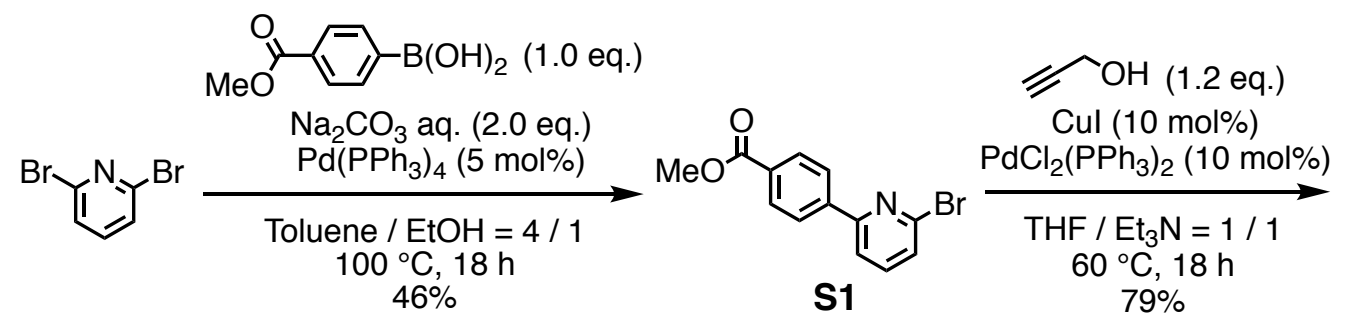<smiles>COC(=O)c1ccc(-c2ccc(C#CCOCC(=O)O)nc2)cc1</smiles>

Scheme S1. Synthesis of 2

\section{Synthesis of S1}<smiles>COC(=O)c1ccc(-c2cccc(Br)n2)cc1</smiles>

$\mathrm{Na}_{2} \mathrm{CO}_{3}$ aq. (1.6 g, $\left.15 \mathrm{mmol}, \mathrm{H}_{2} \mathrm{O} 0.5 \mathrm{~mL}\right)$ was added to a solution of 4-methoxycarbonyl phenylboronic acid (1.3 g, $7.4 \mathrm{mmol})$ and 2,6-dibromopyridine $(1.7 \mathrm{~g}, 7.4 \mathrm{mmol})$ in the mixture of Toluene / $\mathrm{MeOH}=4 / 1(24 \mathrm{~mL})$, then the system was freeze degassed. $\mathrm{Pd}\left(\mathrm{PPh}_{3}\right)_{4}(0.49 \mathrm{~g}, 0.40 \mathrm{mmol})$ was added to a solution quickly, and the mixture was 
stirred for 18 hours at $100{ }^{\circ} \mathrm{C}$. The mixture was extracted with $\mathrm{CH}_{2} \mathrm{Cl}_{2}$, dried by $\mathrm{MgSO}_{4}$, and purified by silica gel column chromatography (eluent; $\left.\mathrm{CH}_{2} \mathrm{Cl}_{2} / \mathrm{Hexane}=1 / 4\right)$ to give $\mathbf{S 1}(0.99 \mathrm{~g}, 46 \%)$ as a white solid; ${ }^{1} \mathrm{H}$ NMR $(300$ $\left.\mathrm{MHz}, 298 \mathrm{~K}, \mathrm{CDCl}_{3}\right) \delta 8.17-8.04(\mathrm{~m}, 4 \mathrm{H}), 7.75\left(\mathrm{dt}, J_{1}=1.0 \mathrm{~Hz}, J_{2}=7.7 \mathrm{~Hz}, 1 \mathrm{H}\right), 7.64\left(\mathrm{td}, J_{1}=1.0 \mathrm{~Hz}, J_{2}=7.7 \mathrm{~Hz}\right.$, $1 \mathrm{H}), 7.47\left(\mathrm{dt}, J_{1}=1.0 \mathrm{~Hz}, J_{2}=7.7 \mathrm{~Hz}, 1 \mathrm{H}\right), 3.95(\mathrm{~s}, 3 \mathrm{H}) \mathrm{ppm}$.

\section{Synthesis of S2}<smiles>COC(=O)c1ccc(-c2cccc(C#CCO)n2)cc1</smiles>

Propargylalcohol $(0.25 \mathrm{~mL}, 4.2 \mathrm{mmol})$ was added to a solution of $\mathbf{S 1}(0.99 \mathrm{~g}, 3.4 \mathrm{mmol})$ and $\mathrm{CuI}(90 \mathrm{mg}, 0.50 \mathrm{mmol})$ in the mixture of THF / $\mathrm{Et}_{3} \mathrm{~N}=1 / 1(10 \mathrm{~mL})$, then the system was freeze degassed. $\mathrm{PdCl}_{2}\left(\mathrm{PPh}_{3}\right)_{2}(0.17 \mathrm{~g}, 0.20 \mathrm{mmol})$ was added to a solution quickly, and the mixture was stirred for 18 hours at $60{ }^{\circ} \mathrm{C}$. The mixture was filtered with Celite, and purified by silica gel column chromatography (eluent; EtOAc / Hexane $=2 / 1)$ to give $\mathbf{S 2}(0.72 \mathrm{~g}, 79 \%)$ as a white solid; ${ }^{1} \mathrm{H}$ NMR $\left(300 \mathrm{MHz}, 298 \mathrm{~K}, \mathrm{CDCl}_{3}\right) \delta 8.17-8.04(\mathrm{~m}, 4 \mathrm{H}), 7.80-7.72(\mathrm{~m}, 2 \mathrm{H}), 7.44\left(\mathrm{dd}, J_{1}=1.7 \mathrm{~Hz}\right.$, $\left.J_{2}=6.9 \mathrm{~Hz}, 1 \mathrm{H}\right), 4.56(\mathrm{~d}, J=6.3 \mathrm{~Hz}, 2 \mathrm{H}), 3.95(\mathrm{~s}, 3 \mathrm{H}), 1.81(\mathrm{t}, J=6.3 \mathrm{~Hz}, 1 \mathrm{H}) \mathrm{ppm}$.

\section{Synthesis of S3}<smiles>O=C(O)c1ccc(-c2cccs2)cc1</smiles>

$\mathrm{NaOH}$ aq. (0.56 g, $\left.14 \mathrm{mmol}, \mathrm{H}_{2} \mathrm{O} 3 \mathrm{~mL}\right)$ was added to a solution of $\mathbf{S 2}(0.70 \mathrm{~g}, 2.6 \mathrm{mmol})$ in the mixture of THF / $\mathrm{MeOH}=3 / 1(15 \mathrm{~mL})$, and the mixture was stirred for 18 hours at room temperature. The solvent was evaporated, water was added to the residue, and washed by $\mathrm{CH}_{2} \mathrm{Cl}_{2} .3 \mathrm{M} \mathrm{HCl}$ aq. was added to the water phase to precipitate the acid, collected the solid, and dried in vacuo to give $\mathbf{S 3}(0.60 \mathrm{~g}, 92 \%)$ as a brown solid; ${ }^{1} \mathrm{H} \mathrm{NMR}(300 \mathrm{MHz}, 298 \mathrm{~K}$, DMSO-d $\left._{6}\right) \delta 8.18(\mathrm{~d}, J=8.6 \mathrm{~Hz}, 2 \mathrm{H}), 8.09-7.98(\mathrm{~m}, 3 \mathrm{H}), 7.91(\mathrm{t}, J=7.7 \mathrm{~Hz}, 1 \mathrm{H}), 7.47(\mathrm{~d}, J=7.7 \mathrm{~Hz}, 1 \mathrm{H}), 4.37(\mathrm{~s}$, 2H) ppm.

\section{Synthesis of 2}<smiles>NC(=O)c1ccc(-c2cccc(C=CCO)n2)cc1</smiles>

Diphenylphosphorylazide (1.3 g, $4.7 \mathrm{mmol})$ was added to a solution of $\mathbf{S 3}(0.79 \mathrm{~g}, 3.1 \mathrm{mmol})$ and DIPEA $(1.2 \mathrm{~g}, 9.3$ $\mathrm{mmol})$ in THF $(20 \mathrm{~mL})$, then, the mixture was stirred at room temperature for 18 hours. To the mixture, sat. $\mathrm{NH}_{4} \mathrm{Cl}$ aq. was added, extracted with $\mathrm{CH}_{2} \mathrm{Cl}_{2}$, washed with water, and purified by silica gel column chromatography (eluent; EtOAc / Hexane $=1 / 1)$ to give $2(0.70 \mathrm{~g}, 80 \%)$ as a white solid; ${ }^{1} \mathrm{H}$ NMR $\left(300 \mathrm{MHz}, 298 \mathrm{~K}, \mathrm{DMSO}-\mathrm{d}_{6}\right) \delta 8.26(\mathrm{~d}$, $J=8.4 \mathrm{~Hz}, 2 \mathrm{H}), 8.12-8.03(\mathrm{~m}, 3 \mathrm{H}), 7.96(\mathrm{t}, J=7.7 \mathrm{~Hz}, 1 \mathrm{H}), 7.54(\mathrm{~d}, J=7.7 \mathrm{~Hz}, 1 \mathrm{H}), 5.46(\mathrm{t}, J=6.0 \mathrm{~Hz}, 1 \mathrm{H}), 4.37$ $(\mathrm{d}, J=6.0 \mathrm{~Hz}, 2 \mathrm{H}) \mathrm{ppm}$; IR (KBr) $v 3422,3221,2922,2853,2171,1686,1264 \mathrm{~cm}^{-1}$.

\section{Synthesis of P7}

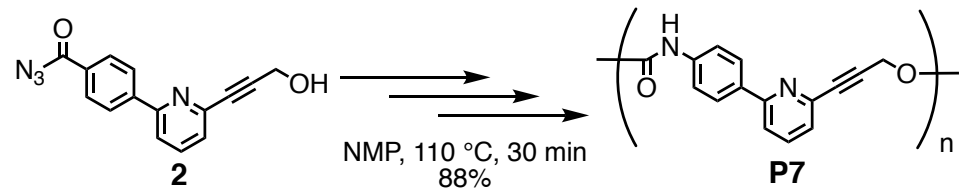

A solution of $2(0.10 \mathrm{~g}, 0.36 \mathrm{mmol})$ in NMP $(0.4 \mathrm{~mL})$ was stirred for $30 \mathrm{~min}$ at $110^{\circ} \mathrm{C}$. The resulting mixture was 
poured into $\mathrm{MeOH}$ and collected the precipitate. The residue was dried in vacuo to give $\mathbf{P 7}(90 \mathrm{mg}, 88 \%)$ as a light glay solid; $M_{\mathrm{n}, \mathrm{SEC}} 3400 \mathrm{~g} / \mathrm{mol} ; M_{\mathrm{w}} / M_{\mathrm{n}} 2.3 ;{ }^{1} \mathrm{H}$ NMR $\left(300 \mathrm{MHz}, 298 \mathrm{~K}, \mathrm{DMSO}-\mathrm{d}_{6}\right) \delta 10.18(\mathrm{~s},(1 \times \mathrm{n}) \mathrm{H}), 8.99(\mathrm{~s}, 1 \mathrm{H})$, $8.03(\mathrm{~d}, J=8.5 \mathrm{~Hz},(2 \times \mathrm{n}) \mathrm{H}), 7.97-7.80(\mathrm{~m},(2 \times \mathrm{n}) \mathrm{H}), 7.62(\mathrm{~d}, J=8.5 \mathrm{~Hz},(2 \times \mathrm{n}) \mathrm{H}), 7.50-7.37(\mathrm{~m},(1 \times \mathrm{n}) \mathrm{H})$, $5.49(\mathrm{t}, J=6.0 \mathrm{~Hz}, 1 \mathrm{H}), 5.12(\mathrm{~s},(2 \times \mathrm{n}) \mathrm{H}), 4.35(\mathrm{~d}, J=6.0 \mathrm{~Hz}, 2 \mathrm{H}) \mathrm{ppm}$.

\section{Synthesis of P8}
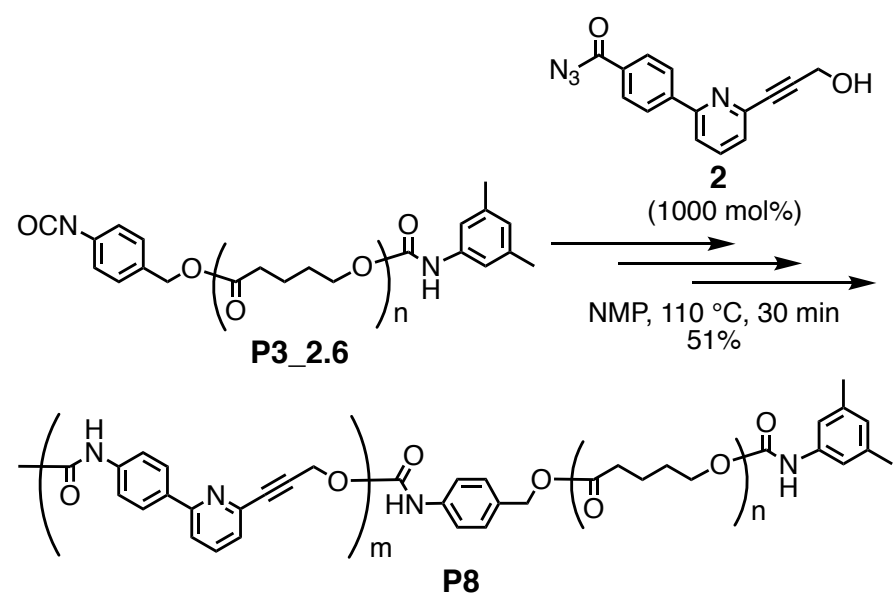

A solution of $2(56 \mathrm{mg}, 0.20 \mathrm{mmol})$ and P3_2.6 (52 $\mathrm{mg}, 0.020 \mathrm{mmol})$ in NMP $(0.20 \mathrm{~mL})$ was stirred for $30 \mathrm{~min}$ at $110{ }^{\circ} \mathrm{C}$. The resulting mixture was poured into $\mathrm{CHCl}_{3}$ and collected the precipitate. The residue was dried in vacuo to give P8 (55 mg, 51\%) as a light brown solid; $M_{\mathrm{n}, \mathrm{SEC}} 4500 \mathrm{~g} / \mathrm{mol} ; M_{\mathrm{w}} / M_{\mathrm{n}} 1.6 ;{ }^{1} \mathrm{H}$ NMR $(300 \mathrm{MHz}, 298 \mathrm{~K}$, DMSO$\left.\mathrm{d}_{6}\right) \delta 10.18(\mathrm{~s},(1 \times \mathrm{n}) \mathrm{H}), 8.03(\mathrm{~d}, J=8.5 \mathrm{~Hz},(2 \times \mathrm{m}) \mathrm{H}), 7.98-7.82(\mathrm{~m},((2 \times \mathrm{m})+2) \mathrm{H}), 7.62(\mathrm{~d}, J=8.5 \mathrm{~Hz},(2 \times$ m) H), $7.46(\mathrm{~d}, J=7.0 \mathrm{~Hz},(1 \times \mathrm{m}) \mathrm{H}), 7.26(\mathrm{~d}, J=7.0 \mathrm{~Hz}, 2 \mathrm{H}), 7.07(\mathrm{~s}, 2 \mathrm{H}), 6.61(\mathrm{~s}, 1 \mathrm{H}), 5.22-5.00(\mathrm{~m},(2 \times \mathrm{m}) \mathrm{H})$, $4.35(\mathrm{~d}, J=6.0 \mathrm{~Hz}, 2 \mathrm{H}), 3.41-3.28\left(\mathrm{~m},(2 \times \mathrm{n}) \mathrm{H},-\mathrm{CH}_{2} \mathrm{O}-\right), 2.39-2.23\left(\mathrm{~m},(2 \times \mathrm{n}) \mathrm{H},-\mathrm{COCH}_{2}-\right), 2.19(\mathrm{~s}, 6 \mathrm{H}), 1.65-$ $1.45\left(\mathrm{~m},((2 \times \mathrm{n})+(2 \times \mathrm{n})) \mathrm{H},-\mathbf{C H}_{2} \mathrm{CH}_{2} \mathrm{O}-\right.$ and $\left.-\mathrm{COCH}_{2} \mathbf{C H}_{2}-\right)$, ppm. 


\section{Spectra of synthesized compounds}

Spectra data not shown on the main text nor previous part of this ESI are listed here.

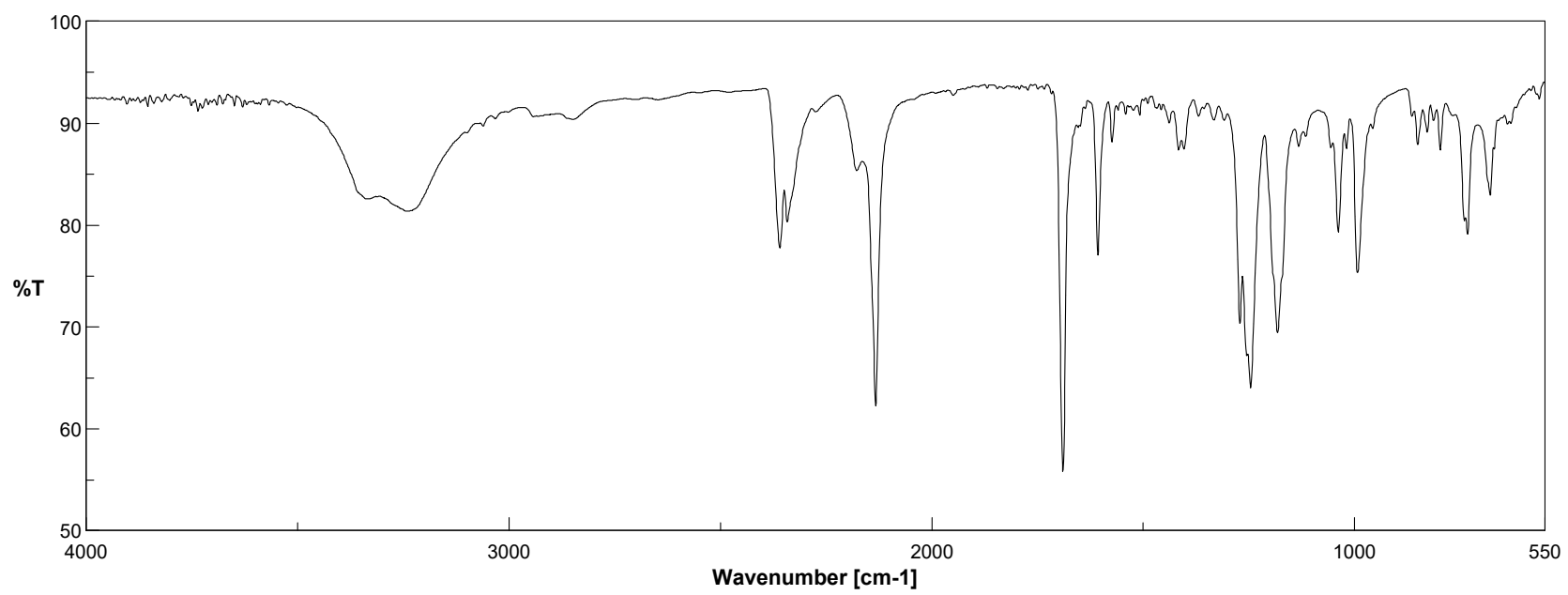

IR spectrum of $\mathbf{1}(\mathrm{NaCl})$.
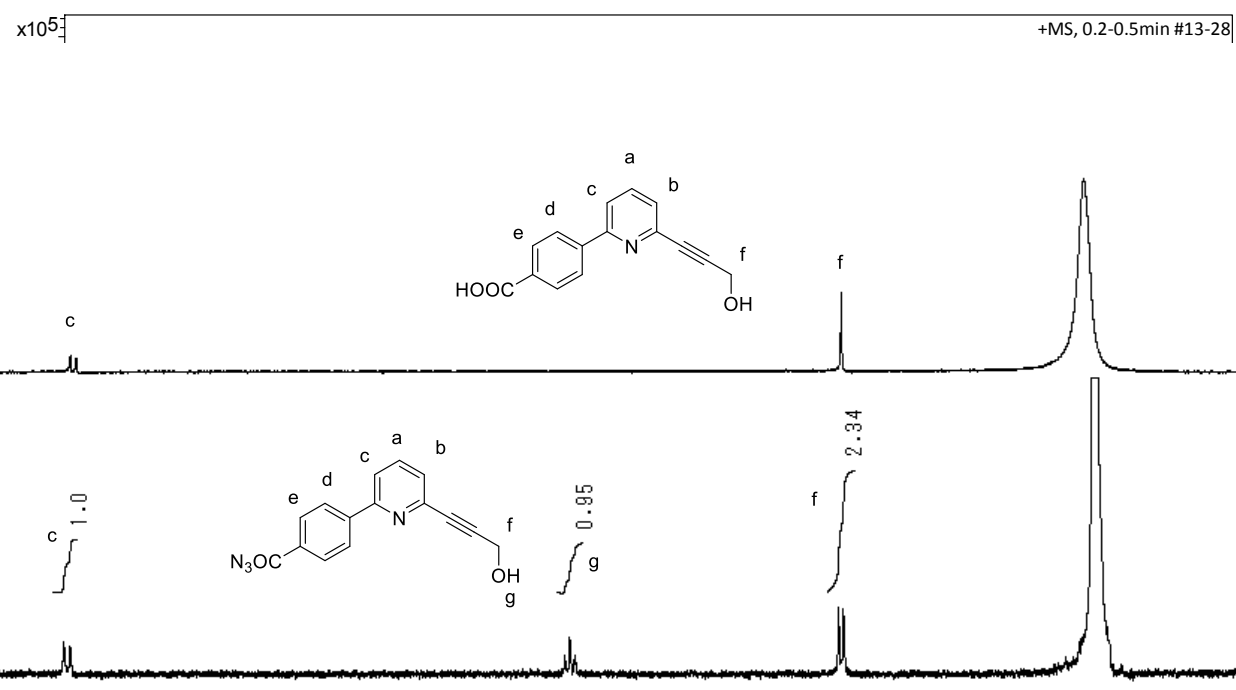

ESI-TOF MS of 1 (upper figure: measured peaks, under figure: calculated peaks)

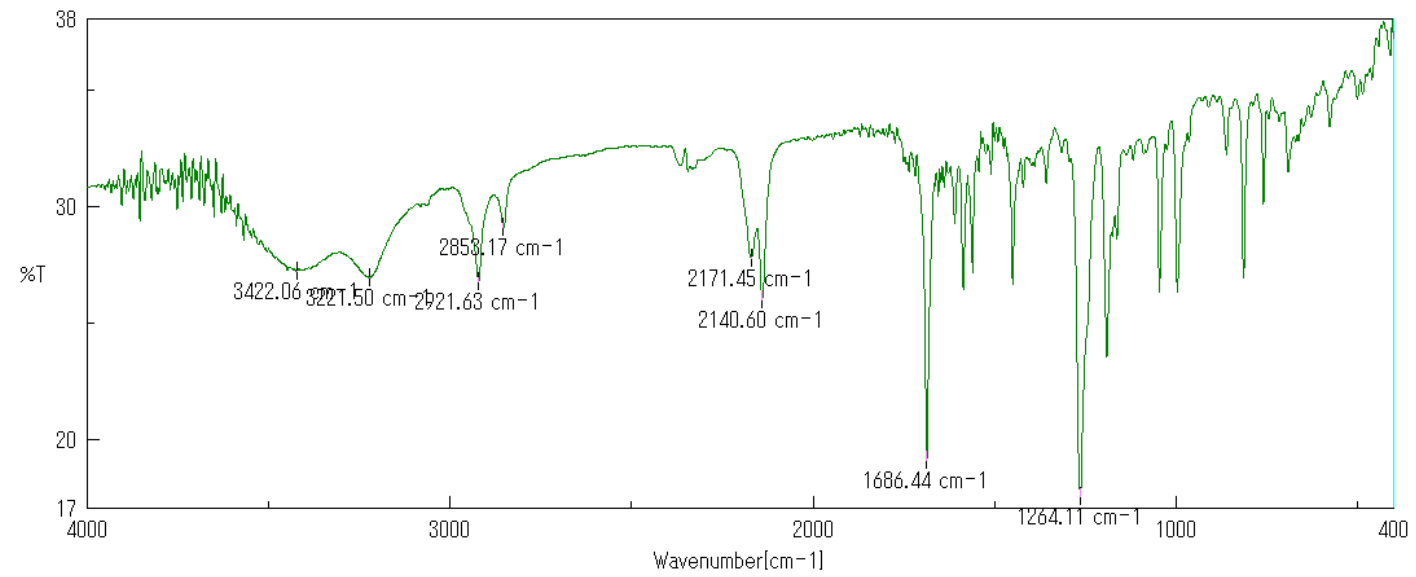

IR spectrum of $2(\mathrm{KBr})$. 


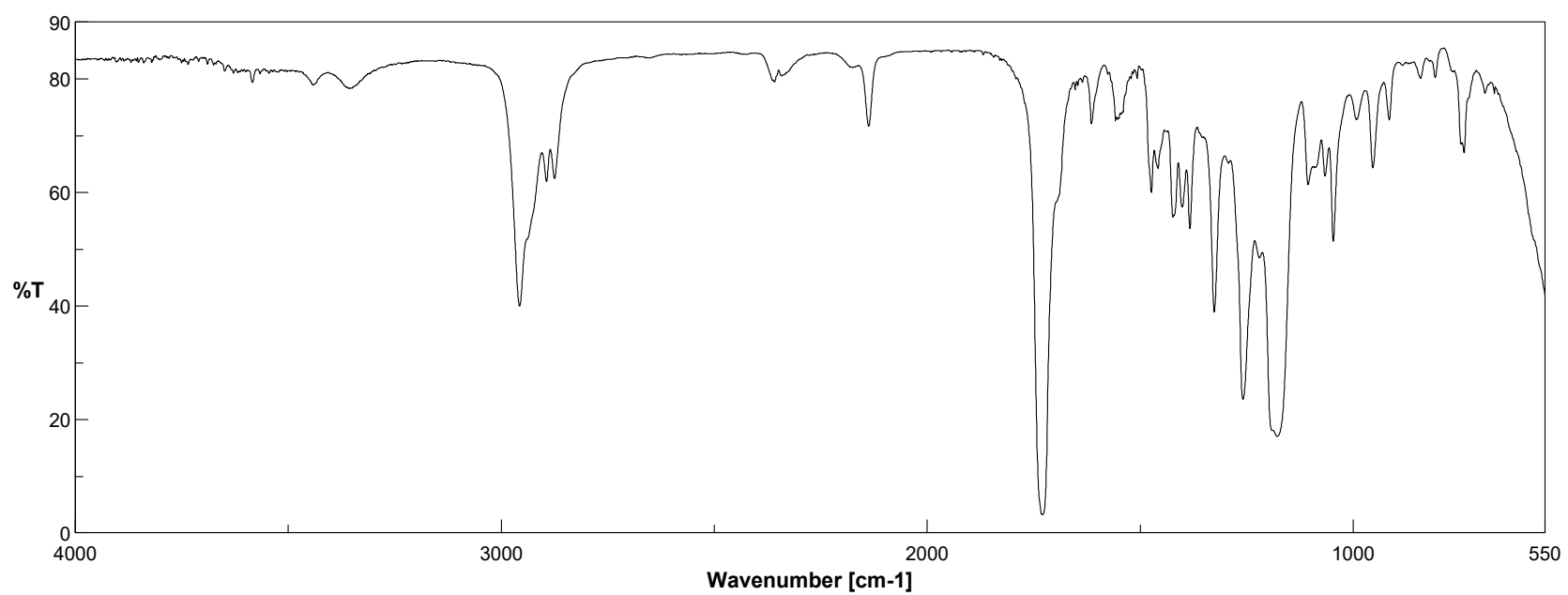

IR spectrum of $\mathbf{P 2}(\mathrm{NaCl})$.

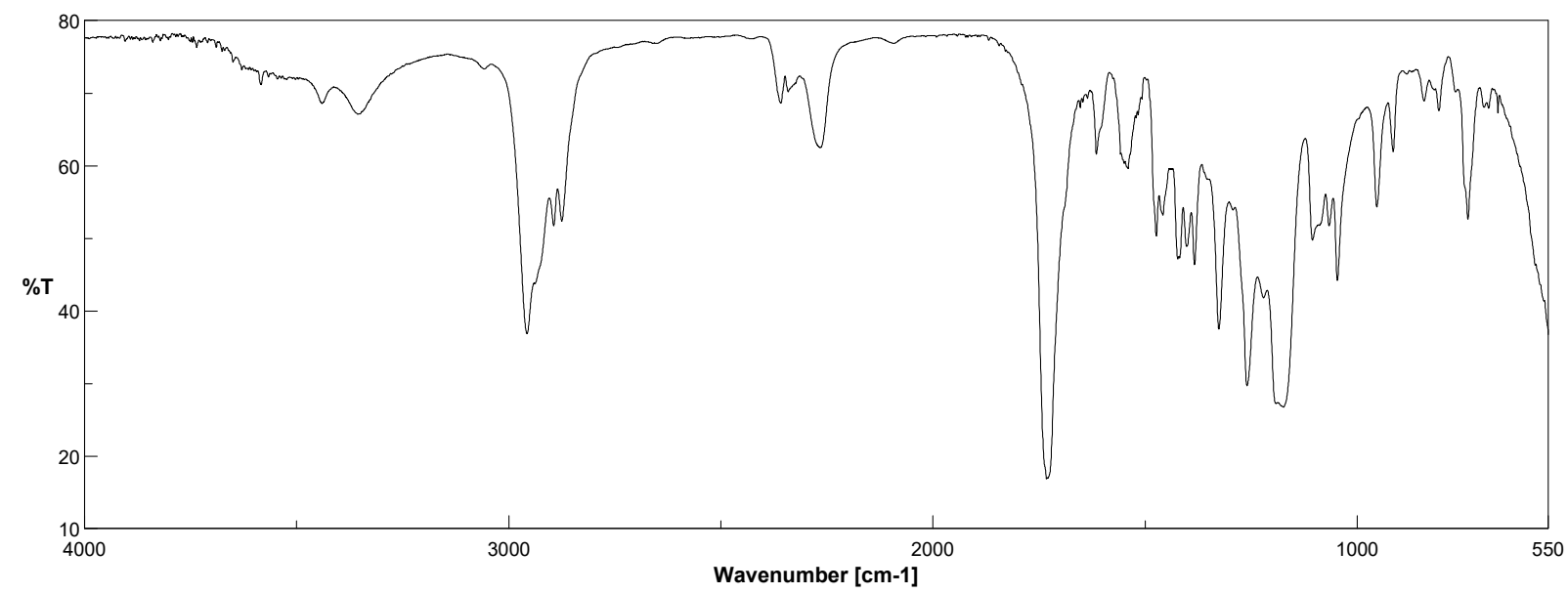

IR spectrum of $\mathbf{P 3}(\mathrm{NaCl})$. 


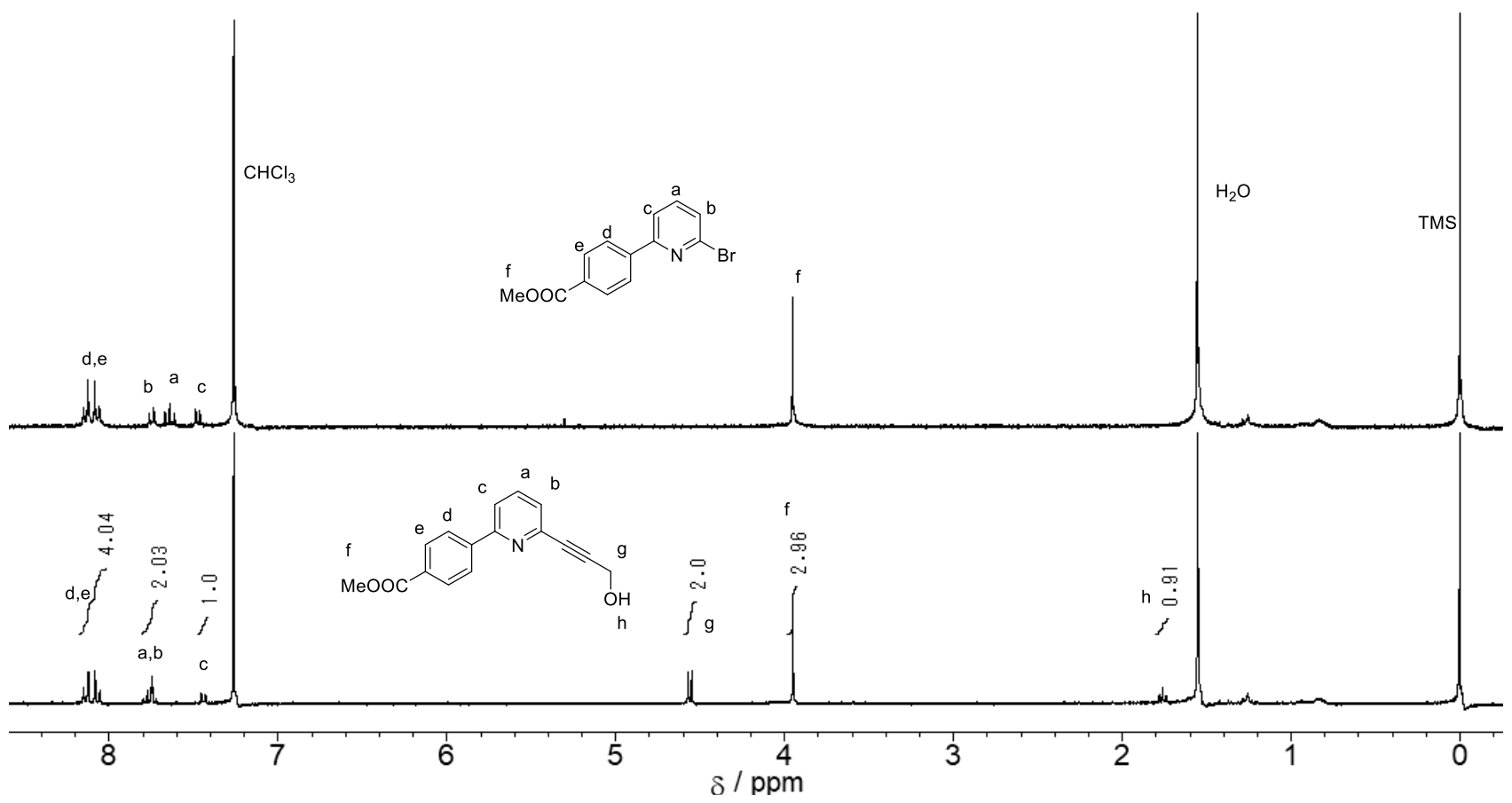

${ }^{1} \mathrm{H}$ NMR spectra of $\mathbf{S 1}$ (top line) and $\mathbf{S 2}$ (bottom line) (300 MHz, $298 \mathrm{~K}, \mathrm{CDCl}_{3}$ )

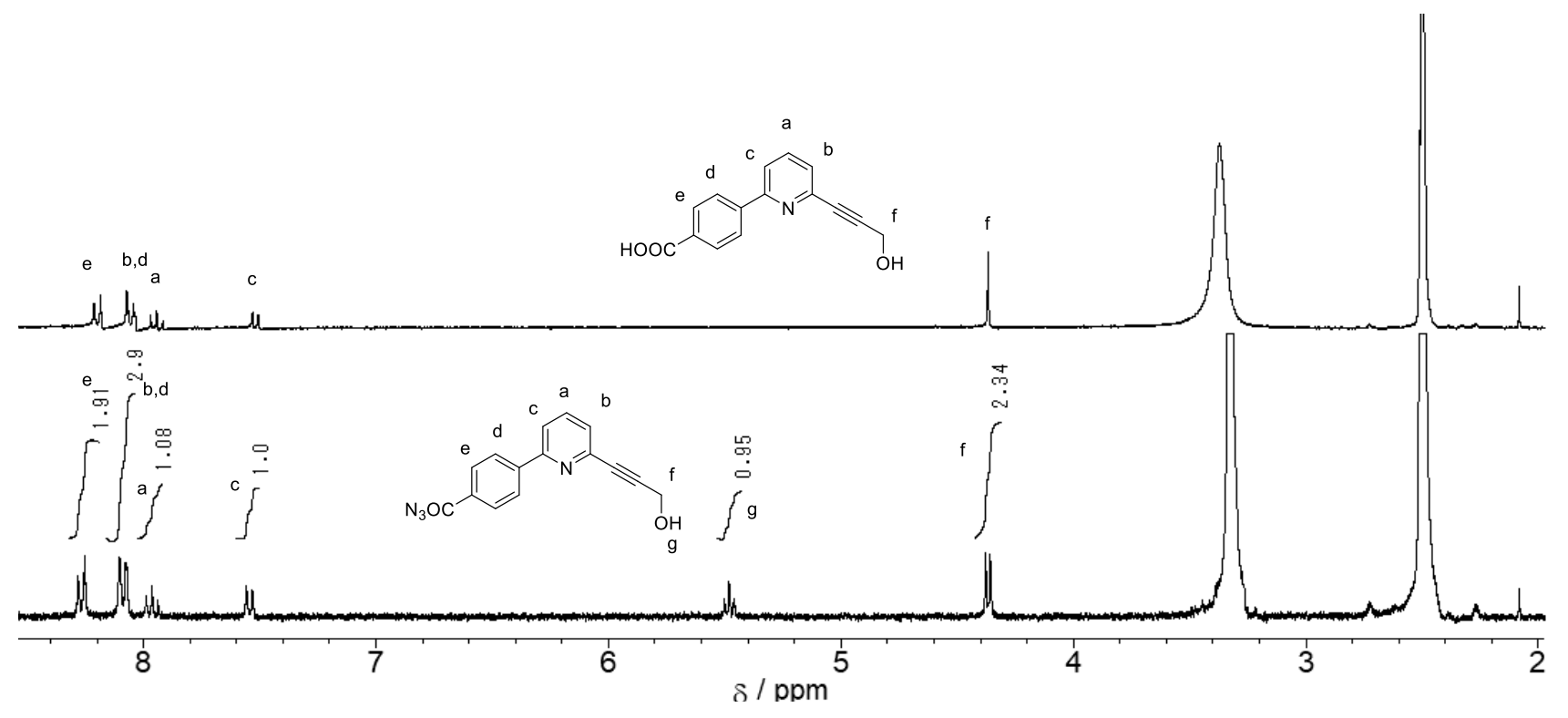

${ }^{1} \mathrm{H}$ NMR spectra of $\mathbf{S 3}$ (top line) and 2 (bottom line) (300 MHz, $298 \mathrm{~K}$, DMSO-d 6 ) 


\section{References}

(1) Y. Akae, H. Sogawa, T. Takata, Eur. J. Org. Chem. 2019, 3605.

(2) A. Sagi, R. Weinstain, N. Karton, D. Shabat, J. Am. Chem. Soc. 2008, 130, 5434.

(3) K. Makiguchi, K. Satoh, T. Kakuchi, Macromolecules 2011, 44, 1999. 